\title{
Comparison of three-compartment Petri dishes and individual plates for routine culture of vaginal
} swabs

\author{
J C Reeder, A P Shakespeare, C Bryan, M G L Keaney, L A Ganguli
}

\begin{abstract}
Recovery of aerobes and facultative anaerobes from 200 consecutive randomly selected high vaginal swabs was evaluated using three-compartment Petri dishes containing Sabouraud dextrose agar, GC selective agar, and chocolate agar. The method was compared with the traditional method using individual Petri dishes. The two methods produced comparable results both in terms and quantities of organisms recovered from the specimens. As threecompartment Petri dishes use less agar, save time in culturing specimens, yet still maintain the same standard of culture, they provide a more economical alternative to the traditional method for routine culture of vaginal swabs.
\end{abstract}

Most vaginal swabs received by hospital laboratories require routine investigations for common pathogens associated with specific vaginitis or bacterial vaginosis. These include Trichomonas vaginalis, yeasts, Staphyococcus aureus, coliforms, $\beta$-haemolytic streptococci, Neisseria gonorrhoeae, Gardnerella vaginalis and other anaerobes. ${ }^{1-3}$ The swabs also carry large numbers of commensal flora, the most prevalent of which are lactobacilli, Staphylococcus epidermidis, diphtheroids, $\alpha$ haemolytic and non-haemolytic streptococi. ${ }^{4}$ Microbiological detection of such a wide range of species requires aerobic and anaerobic culture in addition to microscopic examinations. Routine isolation of aerobes and facultative anaerobes alone utilises at least three different laboratory media, usually Sabouraud agar, GC selective agar, and chocolate agar. These are traditionally contained in individual Petri dishes which necessitates three separate inoculations. The development of Petri dishes divided into compartments of equal size, however, has allowed different types of agar to be contained within the same plate, providing they require the same incubation conditions. This facilitates a single inoculation using a rotary plater which saves time and as the quantities of media required are less, further economies can be made in use of materials.

The aim of this study was to evaluate the use of Petri dishes divided into three compartments containing Sabouraud, GC selective, and chocolate agars for the routine isolation of aerobes and facultative anaerobes from high vaginal swabs. Recovery of both commensal and pathogenic organisms using threecomponent Petri dishes were compared with the traditional method of using individual agar plates.

\section{Methods}

Sabouraud dextrose agar (Lab $M$ ) and chocolate agar using Columbia agar base (Lab M) were prepared according to the manufacturer's instructions. GC selective agar was prepared using GC agar base (Lab $M$ ) supplemented with four GC selectatabs VCNT (each containing vancomycin, colistin, nystatin and trimethoprim) (Mast) and 7\% lysed horse blood. The media were distributed into triplevented three-compartment Petri dishes (Northern Media Ltd) which were divided into segments by half-depth walls. Plates contained $6 \mathrm{ml}$ of each agar, a volume which reached the full height of the dividing wall but ensured that media in adjacent compartments did not touch (figure). The media were also dispensed in $20 \mathrm{ml}$ volumes into individual Petri dishes. Two hundred consecutive, randomly selected high vaginal swabs were used for the study and each was first inoculated over the whole agar surface of a three-compartment plate using a rotary plater. The swabs were then cultured on the media contained in individual plates, covering quarter segments of Sabouraud agar and GC selective agar and then spread with a loop from the original inoculation site over the surface of a whole chocolate agar plate. A $5 \mu \mathrm{g}$ chloramphenicol disc (Oxoid) was placed on Sabouraud agar cultures. All three-compart-

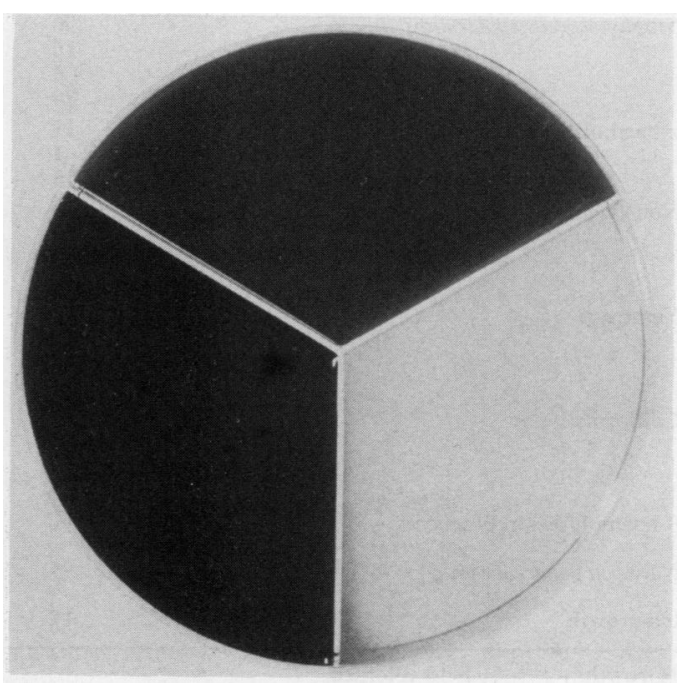

Three-compartment Petri dish containing Sabouraud, $G C$ selective, and chocolate agars.
Mr C Bryan

Accepted for publication 11 June 1990
Microbiology, Hope Mospital, Universit M6 8HD

A P Shakespeare Bryan

M G L Keaney

L A Ganguli 
ment, individual chocolate agar and individual GC selective agar plates were incubated in an atmosphere of $95 \%$ air: $5 \% \mathrm{CO}_{2}$ for 48 hours. Individual Sabouraud agar plates were incubated aerobically. Growth of all organisms on both sets of plates was identified and quantified; numbers of lactobacilli, streptococci, staphylococci and coliforms were estimated from chocolate agar, yeasts from Sabouraud agar, and Neisseria gonorrhoeae from GC selective agar. The amount of growth was recorded semiquantitatively; one to 10 colonies per plate was considered to be a + growth, 10-20 colonies a ++ growth, and more than 20 colonies +++ growth. All swabs were also cultured on neomycin blood agar for anaerobic incubation, and microscopic preparations were examined for the presence of Trichomonas vaginalis and "clue cells" associated with Gardnerella vaginalis and anaerobes. ${ }^{3}$

\section{Results}

Growth of pathogenic and commensal organisms from the 200 high vaginal swabs cultured on media contained in three-compartment plates and individual plates is compared in table 1. Generally, the organisms recovered from a specimen were the same using either method as shown by the total numbers of specimens growing an organism (table 1). For some organisms, however, there was a difference between the methods in the quantities estimated, but no trend towards high or low estimates could be attributed to either method. Growth from each specimen was summarised as for the laboratory report (table 2) and this proved to be identical for each method. One

Table 1 Comparison of quantities of different organisms recovered from media contained in three-compartment and individual Petri dishes

\begin{tabular}{|c|c|c|c|}
\hline \multirow[b]{2}{*}{ Organism } & \multirow[b]{2}{*}{ Growth $h^{\star}$} & \multicolumn{2}{|c|}{ Number of high vaginal swabs } \\
\hline & & $\begin{array}{l}\text { Individual } \\
\text { plates }\end{array}$ & $\begin{array}{l}\text { Three-compartment } \\
\text { plates }\end{array}$ \\
\hline Lactobacillus spp & $\begin{array}{l}+ \\
++ \\
++ \\
+ \\
\text { Total }\end{array}$ & $\begin{array}{l}29 \\
47 \\
13 \\
89\end{array}$ & $\begin{array}{l}33 \\
43 \\
15 \\
91\end{array}$ \\
\hline Staphylococcus epidermidis & $\begin{array}{l}+ \\
++ \\
+++ \\
\text { Total }\end{array}$ & $\begin{array}{r}50 \\
16 \\
4 \\
70\end{array}$ & $\begin{array}{r}45 \\
19 \\
2 \\
66\end{array}$ \\
\hline$x$-haemolytic streptococci & $\begin{array}{l}+ \\
+ \\
\text { Total }\end{array}$ & $\begin{array}{r}11 \\
4 \\
15\end{array}$ & $\begin{array}{r}12 \\
3 \\
15\end{array}$ \\
\hline Non-haemolytic streptococci & $\begin{array}{l}+ \\
++ \\
++ \\
\text { Total }\end{array}$ & $\begin{array}{r}19 \\
30 \\
0 \\
49\end{array}$ & $\begin{array}{r}24 \\
26 \\
1 \\
51\end{array}$ \\
\hline Yeast spp & $\begin{array}{l}+ \\
++ \\
+++ \\
\text { Total }\end{array}$ & $\begin{array}{r}11 \\
17 \\
8 \\
36\end{array}$ & $\begin{array}{r}9 \\
20 \\
7 \\
36\end{array}$ \\
\hline Coliforms + & $\begin{array}{l}+ \\
++ \\
+++ \\
+ \text { Total }\end{array}$ & $\begin{array}{r}15 \\
16 \\
5 \\
36\end{array}$ & $\begin{array}{r}14 \\
15 \\
7 \\
36\end{array}$ \\
\hline$\beta$-haemolytic streptococci & ++ & 1 & 1 \\
\hline Neisseria gonorrhoeae. & ++ & 3 & 3 \\
\hline No growth & & 14 & 14 \\
\hline
\end{tabular}

Table 2 Summary of organisms reported from high vaginal swabs

\begin{tabular}{|c|c|}
\hline Organism & Number of specimens \\
\hline Commensal flora ${ }^{\star}$ & 132 \\
\hline Calbicans & 29 \\
\hline Yeast spp & 7 \\
\hline Coliforms $\dagger$ & 13 \\
\hline N gonorrhoeae & 3 \\
\hline$S$ milleri & 1 \\
\hline Group B Streptococcus & 1 \\
\hline No growth & 14 \\
\hline
\end{tabular}

*Including lactobacilli, $S$ epidermidis, $\beta$-haemolytic streptococci, non-haemolytic streptococci and mixed coliforms.

tpure growth.

hundred and thirty two specimens grew commensal flora only, 54 specimens yielded a single pathogenic isolate, and there was no growth from 14 specimens. Twenty nine of the 36 yeasts isolated were subsequently identified as Candida albicans and one of the $\alpha$-haemolytic streptococci as Streptococcus milleri.

\section{Discussion}

Three-compartment Petri dishes containing Sabouraud, GC selective, and chocolate agars proved a suitable alternative to traditional individual plates for routine detection of aerobes and facultative anaerobes from high vaginal swabs. Numbers and types of both commensal and pathogenic organisms were comparable using either method, and between them the 200 specimens examined yielded the majority of the organisms expected. With the continuing desire to reduce hospital laboratory costs, the three-compartment plates are more economical in terms of both time and materials. Time is saved by the requirement of only one inoculation and in the labelling of plates, and although three-compartment plates are more expensive than conventional Petri dishes, they use less agar. Culture of high vaginal swabs for aerobes and facultative anaerobes using threecompartment plates has been calculated at $£ 0.36$ a specimen compared with $£ 0.54$ using the traditional method (1990 prices) - a saving of $33 \%$. Further advantages of the method are the ease of reading a single plate and saving on storage space both in refrigerators and incubators. Potential drawbacks of the method are that the volume of agar dispensed into the sections of the plates must be accurately measured because it has to be of sufficient depth to facilitate rotary plating, but not too high so that adjacent agars touch, which may result in diffusion of antibiotics. This is easily overcome by use of an automatic agar dispenser. GC selective and chocolate agars are usually incubated in an increased carbon dioxide atmosphere, but Candida spp and other yeasts normally prefer aerobic conditions. It has been shown, however, that they will grow under increased concentrations of carbon dioxide in air, ${ }^{5}$ and no difference was observed in the total numbers isolated from either atmosphere in this study.

Three-compartment plates have been successfully used for culturing 20-30 high vaginal swabs daily in this laboratory for eight years. A 
slight modification to the method is that chloramphenicol is now incorporated into Sabouraud agar instead of using discs. The method is also ideal for use in STD clinics. It could also potentially be applied to other specimens which are cultured on different agars requiring the same incubation conditions such as faecal specimens and routine anerobic cultures.
1 De Costa EJ. Infection of the vagina and vulva. Clin Obstet Gynecol 1969;12:198-218.

Aynecol 1969;12:198-218. Amsel R, Totten PA, Spiegel CA
vaginitis. $A m J$ Med 1983;74:14-22.

3 Blackwell AL, Fox AR, Phillips I, et al. Anaerobic vaginosis (non-specific vaginitis): clinical, microbiological and therapeutic findings. Lancet 1983;ii: 1379-82.

4 Bartlett JG, Polk BF. Bacterial flora of the vagina: Quantitative study. Rev Infect Dis 1984;6(suppl 1):S67-S72.
Webster CE, Odds FC. Growth of pathogenic Candid isolates anaerobically and under elevated concentrations of $\mathrm{CO}_{2}$ in air. J Med Vet Mycol 1987;25:47-54. 